\title{
Root exudates from citrus plants subjected to abiotic stress conditions have a positive effect on rhizobacteria
}

\author{
Vicente Vives-Peris ${ }^{1}$, Lázaro Molina ${ }^{2}$, Ana Segura ${ }^{2}$, Aurelio Gómez-Cadenas ${ }^{1}$, $\operatorname{Rosa}^{-}$ \\ María Pérez-Clemente ${ }^{1}$
}

${ }^{1}$ Departament de Ciències Agràries i del Medi Natural, Universitat Jaume I, Avda. Sos Baynat s/n, 12071, Castellón de la Plana, Spain

${ }^{2}$ Environmental Protection Department, Estación Experimental del Zaidín, Consejo Superior de Investigaciones Científicas, C/ Profesor Albareda 1, 18008, Granada, Spain

\begin{abstract}
Plants are constantly releasing root exudates to the rhizosphere. These compounds are responsible of different (positive or negative) interactions with other organisms, including plants, fungi or bacteria. In this work, the effect of root exudates obtained from in vitro cultured citrus plants on two rhizobacteria (Pseudomonas putida KT2440 and Novosphingobium sp. HR1a) was evaluated. Root exudates were obtained from two citrus genotypes differing in their sensitivity to salt and heat stress and affected differentially the growth of both rhizobacteria. Root exudates from salt-stressed plants of $C$. macrophylla (salt tolerant) induced an increase in bacterial growth higher than that obtained from Carrizo citrange exudates (salt sensitive). Root exudates from heat-stressed plants also had a positive effect on bacterial growth, which was more evident in the heatsensitive $C$. macrophylla. These results reveal that the growth of these rhizobacteria can be modulated through citrus root exudates, and it can change depending on the stress conditions and the genotype. Biosensors P. putida KT2442 (pMIS5) and Novosphingobium sp. HR1a (pPAH) were used to test the presence of proline and salicylates in root exudates by measuring $\beta$-galactosidase activity. This activity increased in presence of root exudates obtained from stressed plants, and in a higher extent in the case of exudates obtained from the genotype resistant to each particular stress, indicating that those root exudates contain larger quantities of proline and salicylates, as it has been described previously. Our data reveals that both $P$. putida KT2442 (pMIS5) and Novosphingobium sp. HR1a (pPAH), could be used as biosensors of plant stress.
\end{abstract}


Keywords: citrus, heat stress, rhizobacteria, root exudates, salt stress

Abbreviations: ACC: aminocyclopropane-1-carboxylic acid; 2: Arbuscular mycorrhizal fungi; CFU: Colony forming units; Cm: Chloramphenicol; HLB: Huanglongbing; LB: Lysogeny brot; MS: Murashige and Skoog salt solution; ONPG: ortho-nitrophenyl- $\beta$ galactoside; PGPR: Plant growth promoting rhizobacteria; Rif: Rifampicin; SA: Salicylic acid; Tc: Tetracycline

\section{Introduction}

Plants release between 5 and $25 \%$ of net fixed carbon into the rhizosphere in the form of compounds ranging from simple organic anions to complex polymer mucilages (Bais et al., 2006). Those specialized metabolites, produced and secreted by plants, play a critical role in the interaction of plants with soil organisms at the root vicinity, generally increasing the total quantity and activity of microbes around plant roots. These microorganisms use the compounds released by the plants through their roots as major nutrient sources, mainly carbon and nitrogen, constituents of sugars and amino acids, which are the compounds released to the rhizosphere in higher quantities (Lugtenberg et al., 1999; Moe, 2013). This is the case of the plant growth promoting rhizobacteria (PGPR) Pseudomonas putida, which growth is positively affected by proline, one of the amino acids largely released to the rhizosphere (Vílchez et al., 2000b). In addition, flavonoids, anthocyanins or salicylates, present in root exudates in lower quantities can affect rhizosphere biotic composition (Badri and Vivanco, 2009; Cesco et al., 2012; Wu et al., 2009).

Root exudation is highly influenced by various biotic and abiotic factors in the surrounding environment, which can lead to a significant shift in the rhizosphere microbiota (Kawasaki et al., 2016). Several studies carried out with herbaceous species, reveal that PGPR and arbuscular mycorrhizal fungi (AMF) alleviate the damage induced by abiotic stresses conditions such as drought, salinity, flooding, nutrient deprivation, heavy metal or high temperatures in plants (Ali et al., 2011). The association with the bacteria Scytonema hofmanni alleviates the adverse effects of salt stress in rice plants (Rodríguez et al., 2006). Meanwhile, Burkholderia phytofirmans PsJN improves shoot and root growth and tuberization in potato plants cultured, either in vitro or ex vitro, under heat stress conditions (Bensalim et al., 1998). Wheat plants inoculated with P. putida 
AKMP7 are less damaged by high temperatures than non-inoculated plants, showing higher levels of chlorophylls, sugars, proline, starch, proteins and amino acids, and a reduction in superoxide dismutase, ascorbate peroxidase and catalase activities (Ali et al., 2011). Among the mechanisms responsible of this protection against abiotic stresses are the bacterial production of indole acetic acid or nitric oxide, which stimulate root growth and development and facilitate nutrient fixation; the decrease of plant endogenous content of ethylene by the bacterial 1-aminocyclopropane-1-carboxylic acid (ACC) deaminase activity or by the induction of changes in the root cell wall or membrane, such as the production of biofilms (Dimkpa et al., 2009).

Therefore, understanding the interactions among living organisms in the rhizosphere will have a great value in improving plant resistance to both biotic and abiotic stresses, which will ultimately result in an increase in crop productivity.

There is little information about the colonization of PGPRs in citrus plant rhizosphere. Isolates belonging to genera Burkholderia, Pantoea, Pseudomonas, Bacilli, Painibacillus, and Serratia were found to be associated with citrus roots, being the soil bacteria population different in plants affected by huanglongbing (HLB) (Trivedi et al., 2011). The beneficial effect of $P$. putida in absence of any stress condition has been also described (Chiquito-Contreras et al., 2012). On the other hand, it has been reported that the AMF mitigates some biotic and abiotic stresses, including phytophthora infection (Watanarojanaporn et al., 2011), drought (Wu and Zou 2009), high salinity (Navarro et al. 2014; Satir et al. 2016; Zhang et al. 2017) or chilling (Wu and Zou 2010).

Among citrus genotypes there is a wide variability concerning to their tolerance to different abiotic or biotic stress conditions. Carrizo citrange is salt sensitive and heat stress tolerant, whereas Citrus macrophylla is salt tolerant and heat stress sensitive (Vives-Peris et al., 2017). In addition, our previous work demonstrates that citrus plants are capable to exude proline and salicylates as cinnamic acid or salicylic acid (SA) by their roots. These and other metabolites present in citrus root exudates could affect the growth of different bacteria present in the rhizosphere (Vílchez et al., 2000a; Vives-Peris et al., 2017; Wu et al., 2009).

In this work, the effect of root exudates of two citrus genotypes Carrizo citrange and Citrus macrophylla with contrasting tolerance to salt and heat stress on the bacteria $P$. putida KT2440 and Novosphingobium sp. HR1a has been studied. Although P. putida 
KT2440 has been previously reported as a PGPR (Planchamp et al., 2015), the role of Novosphingobium sp. HR1a as a PGPR is not clear, being limited to other strains of this genus (Zhang et al., 2016).

\section{Materials and Methods}

\subsection{Root exudates obtainment}

Root exudates from in vitro cultured plants of two citrus rootstocks, Carrizo citrange (Citrus sinensis L. Osbeck x Poncirus trifoliata L. Raf.) and Citrus macrophylla Wester, were obtained as described in Vives-Peris et al., (2017).

Seeds of both genotypes were peeled and disinfected for $10 \mathrm{~min}$ in a $0.5 \%$ ( $\mathrm{vol} / \mathrm{vol}$ ) sodium hypochlorite solution containing $0.1 \%$ (vol/vol) Tween-20 wetting agent and rinsed three times with sterile distilled water. Seeds were sown individually in $25 \times 150$ $\mathrm{mm}$ culture tubes with $20 \mathrm{~mL}$ of germination medium (MS) consisting of Murashige and Skoog salt solution (Murashige and Skoog, 1962) and 3\% of sucrose as carbon source. The $\mathrm{pH}$ was set at $5.7 \pm 0.1$ with $0.1 \mathrm{~N} \mathrm{NaOH}$ before autoclaving. The medium was solidified by the addition of $0.9 \%$ agar (Conda, Madrid, Spain). The cultures were maintained at $25{ }^{\circ} \mathrm{C}$, first in darkness for two weeks and two more weeks with a photoperiod of 16 hours and illumination of $150 \mathrm{mmol} \mathrm{m}^{-2} \mathrm{~s}^{-1}$. After that, 30 plants per treatment were transferred to liquid MS medium and roots were pruned in order to favor the development of new roots.

Twenty days after the transference to MS liquid media, plants with a well-developed root system were transferred to the exudation media, composed by sterile deionized water in control and heat-stressed plants. For salt stress treatments, 60 or $90 \mathrm{mM} \mathrm{NaCl}$ was added to the exudation medium. For heat stress, plants were cultured at 30 or $40{ }^{\circ} \mathrm{C}$ with a 16 hours photoperiod. Control and salt-stressed plants were maintained at $25{ }^{\circ} \mathrm{C}$ with the same photoperiod. Medium was collected after ten days of exudation, frozen with liquid nitrogen and stored at $-80{ }^{\circ} \mathrm{C}$. The absence of contaminations in root exudates was tested by culturing a $20 \mu \mathrm{L}$ aliquot in potato dextrose agar medium (Conda, Madrid, Spain).

\subsection{Bacterial strains and plasmids}

To study the effect of root exudates on rhizobacterial growth, $P$. putida strain KT2440 and Novosphingobium sp. strain HR1a were used. For the detection of proline and 
salicylates, $\beta$-galactosidase assays using $P$. putida KT2442 (pMIS5) and Novosphingobium sp. HR1a (pPAH) respectively were performed. In pMIS5 (Vílchez et al., 2000a) the $P_{\text {puts }}$ promoter was cloned before the lac $Z$ promoterless gene coding for $\beta$-galactosidase, easily measurable, in plasmid pMP220 (Spaink et al., 1987) and in pPAH the $P_{\text {pahA }}$ promoter was controlling the expression of the 'lacZ gene (Segura et al., 2017). Chloramphenicol $(\mathrm{Cm})$, rifampicin (Rif) and tetracycline were added to the bacterial cultures at final concentrations of 30,10 and $10 \mu \mathrm{g} / \mathrm{mL}$ respectively when it was necessary (Table 1).

\subsection{Bacterial growth assays}

The initial bacterial cultures were maintained overnight in $25 \mathrm{~mL}$ flasks containing 10 $\mathrm{mL}$ of lysogeny broth (LB) liquid medium (Bertani, 1951) at $30^{\circ} \mathrm{C}$ in an orbital shaker at $200 \mathrm{rpm}$. These cultures were washed with sterile distilled water twice and adjusted to an initial $\mathrm{OD}_{660 \mathrm{~nm}}$ of the cultures of 0.1 .

To perform the different experimental approaches, bacteria were cultured in glass tubes with $2 \mathrm{~mL}$ of liquid M8 minimal medium (Kohler et al., 2000) supplemented with $20 \mathrm{mM}$ succinate as carbon source and root exudates at the original concentrations. For this, 15 $\mathrm{mL}$ of root exudates were freeze-dried and resuspended in $1.5 \mathrm{~mL}$ of sterile deionized water, being 10-fold diluted when applied to the bacterial medium. Mocks with bacteria growing in M8 minimal medium containing $20 \mathrm{mM}$ succinate, and supplemented with 60 or $90 \mathrm{mM} \mathrm{NaCl}$ were used to consider the salt effect on the bacteria. Bacterial cultures with root exudates were incubated at $30^{\circ} \mathrm{C}$ in an orbital shaker at $200 \mathrm{rpm}$ during $48 \mathrm{~h}$.

Quantification of bacterial growth was performed both, by assessing OD $_{660 \mathrm{~nm}}$ and by counting the colony forming units (CFU). OD at a wavelength of $660 \mathrm{~nm}$ was recorded with a 96 well microplate spectrophotometer (Sunrise, Tecan, Männedorf, Switzerland). Results were expressed as the variation of $\mathrm{OD}_{660 \mathrm{~nm}}$ in comparison with the respective mock and referred to the root fresh weight (Doornbos et al., 2011; Neal et al., 2012). CFUs were determined by plating appropriate bacterial culture dilutions on LB medium supplemented with $\mathrm{Cm}$ and Rif for P. putida KT2440 and Novosphingobium sp. HR1a cultures respectively. CFUs were counted after $24 \mathrm{~h}$ of incubation at $30^{\circ} \mathrm{C}$ (Goldman and Green, 2008).

\subsection{Induction of the PputA and PpahA promoters by different metabolites}


P. putida KT2442 (pMIS5) and Novosphingobium sp. HR1a (pPAH) were cultured in M9 minimal media plus $20 \mathrm{mM}$ succinate as carbon source in the presence of different chemicals related to proline and salicylate. Specifically, tubes containing $2 \mathrm{~mL}$ of M9 medium plus $20 \mathrm{mM}$ succinate supplemented with L-proline or hydroxyl-L-proline $(0.1$ and $1 \mathrm{mM}$ ) were inoculated with P. putida KT2442 (pMIS5) to an initial OD $_{660 \mathrm{~nm}}$ of 0.1 . Tubes with $2 \mathrm{~mL}$ of M9 medium plus $20 \mathrm{mM}$ of succinate supplemented with 0.1 or $1 \mathrm{mM}$ of different salicylic acid biosynthesis and conjugation pathways compounds (methyl salicylate, sodium salicylate, L-phenylalanine, sodium benzoate, $p$-coumaric acid and $t$ cinnamic acid; Fig. Sup. 1) were inoculated with Novosphingobium sp. HR1a (pPAH) to an initial $\mathrm{OD}_{660 \mathrm{~nm}}$ of 0.1 . Tubes were cultured at $30{ }^{\circ} \mathrm{C}$ in an orbital shaker and $\beta$ Galactosidase activity was measured after 7 and 24 hours as in Miller (1972).

\subsection{Induction of the PputA and PpahA promoters by root exudates}

$\beta$-Galactosidase activity was measured in $P$. putida KT2442 (pMIS5) and Novosphingobium sp. HR1a (pPAH) bacteria cultured in M8 minimal medium supplemented with $20 \mathrm{mM}$ succinate and root exudates. $\beta$-Galactosidase activity was determined as described in Miller (1972). Finally, results of $\beta$-galactosidase activity were calculated in Miller units, and the results were normalized according to the root fresh weight.

\subsection{Statistical analyses}

Statistical analyses were assessed with the Statgraphics Plus v.5.1. Software (Statistical Graphics Corp., Herndon, VA, USA). Data are means of three independent replications and were subjected to one- or two-way analysis of variance (ANOVA) and a Tukey posthoc test $(\mathrm{p} \leq 0.05)$ when statistical significant differences were detected.

\section{Results}

\subsection{Bacterial tolerance to saline conditions}

The addition of $\mathrm{NaCl}$ had a negative effect on the turbidity of cultures of both bacterial strains. After 24 hours, $P$. putida KT2440 showed to be more sensitive to saline stress, being unable to reach the $\mathrm{OD}$ recorded in absence of $\mathrm{NaCl}$ at the used $\mathrm{NaCl}$ concentrations (a decrease about the $35 \%$ with respect to the control without $\mathrm{NaCl}$ at 60 or $90 \mathrm{mM}$ was 
observed). In the case of Novosphingobium sp. HR1a, the severity of the imposed stress (60 or $90 \mathrm{mM} \mathrm{NaCl}$ ) conditioned the turbidity in comparison with cultures grown in absence of $\mathrm{NaCl}$. The diminution of the $\mathrm{OD}_{660 \mathrm{~nm}}$ in cultures with $90 \mathrm{mM} \mathrm{NaCl}$ was lower than in the case of P. putida KT2440 being in Novosphingobium sp. HR1a the decrease only of $19.07 \%$ after 24 hours. Moreover, the turbidity of Novosphingobium sp. HR1a cultures was only affected after 24 hours in presence of $90 \mathrm{mM} \mathrm{NaCl}$, whereas the turbidity of the cultures with $60 \mathrm{mM} \mathrm{NaCl}$ was similar to the observed in control conditions (Fig. 1A).

Similar results were observed in the number of CFU after 6 hours of culture, when a decrease of this parameter was observed in cultures of $P$. putida KT2440, with a diminution of CFU values about 52 and 99\% in bacteria grown with 60 and $90 \mathrm{mM}$, respectively, in comparison to control cultures, while no differences were observed in cultures of Novosphingobium sp. HR1a in presence of $\mathrm{NaCl}$ (Fig. 1B).

\subsection{Bacterial growth in presence of root exudates from salt-stressed citrus plants}

Root exudates obtained from Carrizo citrange did not significantly promoted the growth of $P$. putida KT2440 or Novosphingobium sp. HR1a (Fig 2A and 2C); furthermore, the number of $P$. putida KT2440 CFUs decreased with exudates from Carrizo plants stressed with $90 \mathrm{mM} \mathrm{NaCl}$ (Fig 3).

Interestingly, when cultures of the two strains were supplemented with root exudates obtained from salt-stressed Macrophylla plants, a significant increase in turbidity was observed when compared those obtained from non-stressed plants (Fig. 2B and 2D). The increase in turbidity was higher with exudates from plants treated with $90 \mathrm{mM} \mathrm{NaCl}$ than with those from plants treated with $60 \mathrm{mM} \mathrm{NaCl}$, at least during the first 10 hours. The increase in turbidity between the cultures with root exudates from control plants and the culture with root exudates from plants treated with $90 \mathrm{mM} \mathrm{NaCl} 6 \mathrm{~h}$ after inoculation (Fig. 2B) correlates with an increase of two orders of magnitude in the number of $P$. putida KT2440 CFUs (Fig. 3). However, an increase of turbidity of 0.5 OD $_{660 \mathrm{~nm}}$ per gram of root in Novosphingobium sp. HR1a $(\mathrm{t}=6 \mathrm{~h})$ the increase in the number of CFU was only of one order of magnitude (Fig. 3).

\subsection{Bacterial growth in presence of root exudates from heat-stressed citrus plants}


Exudates obtained from plants stressed at $40^{\circ} \mathrm{C}$ were able to support growth of both strains (Fig 4), except exudates from Carrizo plants with Novosphingobium sp. HR1a (Fig. 4C). The effect of the exudates from heat-stressed plants was more evident after 10 hours of growth. Accordingly with the increase in turbidity (Fig. 4), no differences in the number of $P$. putida KT2440 CFUs were detected with exudates from Carrizo plants at 24 hours (Fig 5); while the number of CFUs increase more than one order of magnitude with exudates from Macrophylla plants stressed at $40^{\circ} \mathrm{C}$. At $24 \mathrm{~h}$ the increases in the turbidity observed in Novosphingobium sp. HRla cultures with exudates from both treatments were not high enough as to reflect any increases in the number of CFUs.

\subsection{Differential induction of the $\boldsymbol{P}_{p u t A}$ and $\boldsymbol{P}_{\text {pahA }}$ promoters involved in proline and salicylates detection}

P. putida KT2442 (pMIS5) was previously reported to be able to detect the amino acid proline (Vílchez et al., 2000b) and Novosphingobium sp. HR1a (pPAH) detects salicylate and different polycyclic aromatic hydrocarbons (Segura et al., 2017). As proline, salicylate and related compounds can be found in the rhizosphere, we tested the $\beta$ galactosidase activity of these biosensors in response to different commercial chemicals.

In cultures of $P$. putida KT2442 (pMIS5), $\beta$-galactosidase activity was affected in the presence of L-proline and hydroxy-L-proline. L-proline increased the $\beta$-galactosidase activity around 7 - and 12-fold at 0.1 and $1 \mathrm{mM}$ concentration respectively. Induction with hydroxyl-L-proline was only detectable at $1 \mathrm{mM}$ (almost 3-fold increase) and only after 24 hours (Fig. 6A).

As expected, $\beta$-galactosidase activity of Novosphingobium sp. HR1a (pPAH), was increased by $0.1 \mathrm{mM}$ of sodium salicylate after 24 hours (> 5-fold induction). Interestingly, $0.1 \mathrm{mM}$ methyl salicylate and sodium benzoate were also good inductors of the $\beta$-galactosidase activity $(\approx 3$-fold and 4-fold induction respectively after 7 hours; Fig. $6 \mathrm{~B})$.

These experiments demonstrated that $P$. putida KT2442 (pMIS5) and Novosphingobium sp. HR1a (pPAH) are able to detect compounds present in the medium.

\section{5 $\beta$-Galactosidase activity in presence of citrus root exudates}

Proline, hydroxy-L-proline, salicylate, methyl-salicylate and benzoate were barely detected in Carrizo exudates in presence of exudates from salt-stressed plants, and 
significant increases in $\beta$-galactosidase activity were not detected after 7 hours (Fig. 7). However, these compounds were detected in Macrophylla exudates at higher levels in those obtained from 60 or $90 \mathrm{mM} \mathrm{NaCl}$ stressed plants, reaching 6 and 7-fold increases in P. putida (pMIS5) and 7 and 1.4-fold increase in the case of Novosphingobium sp.

In presence of exudates from heat-stressed plants, $\beta$-galactosidase activity did not show big increases after 24 hours; although there was a tendency toward higher $\beta$-galactosidase activity levels in Carrizo exudates from plants stressed at $40^{\circ} \mathrm{C}$ but no significant differences in the expression of the respective promoters of the biosensors were observed (Fig. 8).

\section{Discussion}

In this work, the effect of root exudates from citrus plants subjected to salt or heat stress on plant rhizobacteria $P$. putida KT2440 and Novosphingobium sp. HR1a has been studied. The work was performed using root exudates from two genotypes differing in their tolerance to salt and heat stress: while Carrizo is a salt-sensitive but heat-tolerant citrus rootstock, Macrophylla had the opposite behavior (salt-stress tolerant and heatsensitive,Vives-Peris et al., 2017). Although the role of $P$. putida as PGPR has been well established in a wide variety of plants as coffee, cocoa or coconut (Kejela et al., 2017; Khadeejath Rajeela et al., 2017), to our knowledge there is no information related the possible effect of Novosphingobium sp. HR1a on plants, although other strains of this genus as Novosphingobium oryzae or Novosphingobium pokkalii have been reported as PGPRs (Krishnan et al., 2017; Zhang et al., 2016).

Our results revealed that citrus root exudates modulate the growth of both rhizobacterial strains when they are grown in a minimal medium without any nitrogen source apart from root exudates, being root exudates from Macrophylla plants subjected to abiotic stress conditions those which promote their growth in a greater extent. The positive effect of plant exudates on bacterial growth can be due to the presence of extra carbon and nitrogen inputs by root exudates, generally as part of sugars and amino acids, as it has been reported by previous studies where root exudates from some plants, such as cucumber or banana, promote growth and biofilm formation of PGPRs Bacillus amyloliquefaciens SQR9 and Bacillus subtilis N11 growth (Zhang et al., 2014). However, growth promotion can be also due to the presence of other metabolites secreted to the rhizosphere in lower concentration, such as hormones or growing factors which are differentially exuded, both 
quantitatively and qualitatively, depending on the citrus genotype (Vives-Peris et al., 2017).

The presence of 60 and $90 \mathrm{mM} \mathrm{NaCl}$ in the culture medium has a detrimental effect in the survival of $P$. putida KT2440 and Novosphingobium sp. HR1a but especially in $P$. putida KT2440 (Fig. 1). Novosphingobium sp. HR1a was originally isolated from seaside soils which commonly present high salt levels, and it has been reported that this bacteria is also able to growth on marine artificial media (Segura et al., 2017). Our results sustained that this strain has developed adaptive mechanisms to tolerate high salinity. These results are in concordance with other works which confirm that size and activity of the soil microbial community is reduced under soil salinity and sodicity induced by irrigation, resulting in a reduction in the soil organic matter decomposition and the mineralization of carbon, nitrogen, sulphur and phosphorus (Rietz and Haynes, 2003).

Therefore, understanding the interaction among plants and microbes under salt stress conditions is of great interest to evaluate the soil quality, especially in agricultural soils of the Mediterranean area. We have observed that exudates from Macrophylla saltstressed plants are able to promote the growth of $P$. putida KT2440 and Novosphingobium sp. HR1a, on the contrary exudates from Carrizo salt-stressed plants did not promote bacterial growth (Figs. 2 and 3). Moreover, in presence of exudates from Macrophylla salt-stressed plants, the promotion of the development in Novosphingobium sp. HR1a was higher than in $P$. putida KT2440, which could be due to the higher tolerance of this strain to high salinity.

Interestingly, in root exudates from the salt-tolerant genotype Macrophylla the amount of proline and/or hydroxyl-L-proline (detected with the biosensor P. putida KT2442 (pMIS5)) and the amount of salicylates (detected with the biosensor Novosphingobium sp. HR1a (pPAH)) is higher than in exudates of Carrizo; furthermore, the induction of the promoters of both biosensors is higher in exudates from Macrophylla plants treated with salt, suggesting a higher exudation of these compounds under salt stress (Fig. 7). Previous studies reported an increase of proline and salicylates content in citrus root exudates from plants subjected to salt stress (Vives-Peris et al., 2017), which is in concordance with the measurements with these biosensors, suggesting that these biosensors could be effectively used to detect exuded proline and salicylates. These compounds can be used as nutrients by soil microorganisms and therefore, the higher growth supported by Macrophylla exudates could be due to the presence of these nutrients that are not present (or present at 
lower levels) in Carrizo exudates. Root exudates from Glycine max (soybean) and Phaesolus vulgaris (common bean), have been also described as promoters of Chryseobacterium balustinum growth (Dardanelli et al. 2010, 2012). However, we cannot exclude the effect of other phytohormones or metabolites present in the exudates of the plant. Previous studies with citrus plants subjected to different abiotic stresses as drought or high temperatures, have demonstrated that tolerant rootstocks have an enhanced antioxidant system (Zandalinas et al., 2017). In addition, tolerant citrus plants subjected to salt stress maintain a higher photosynthetic rate than sensitive plants, being this parameter directly linked with the exudation rate. The exudation of metabolites obtained from the $\mathrm{C}$ fixed in the photosynthesis which could be used as nutrient sources by PGPRs would be increased (Bais et al., 2006; López-Climent et al., 2008). Finally, the higher concentration of antioxidant compounds in tissue which could be related to their higher exudation, promoting the growth of PGPRs and playing an important role in the mutualism among citrus and PGPRs, which could contribute to a better colonization by these bacteria and the alleviation of salt stress to the plant, as it has been reported that $P$. putida and Novosphingobium sp. are able to mitigate the adverse effects of this stress in herbaceous plants (He et al., 2017; Krishnan et al., 2017; Yao et al., 2010).

When root exudates were obtained from heat-stressed plants, those obtained from plants subjected to $30{ }^{\circ} \mathrm{C}$ did not affect the growth of either $P$. putida KT2440 or Novosphingobium sp. HR1a, but root exudates from plants subjected to $40{ }^{\circ} \mathrm{C}$ stimulate growth of both strains, mainly in presence of root exudates from the heat sensitive Macrophylla (Fig. 4). The lack of differences in the growth of both strains in presence of root exudates from plants subjected to $30^{\circ} \mathrm{C}$ may be due to the fact that this temperature is not high enough to induce a severe stress in plants (considering that citrus is a subtropical crop) and consequently it does not affect root exudation as much as salt stress or higher temperatures such as $40^{\circ} \mathrm{C}$ (Vives-Peris et al. 2017). Moreover, the higher sensitivity to this stress condition could explain an earlier (in a lower threshold temperature) modification of root exudate composition in Macrophylla plants. In addition, the higher growth of both strains in presence of exudates from salt-stressed plants in comparison with those obtained from heat-stressed ones can be due to the different character of each adverse condition: whereas salt stress affects first to the root system, originating direct changes in this organ, heat stress affects mainly to the canopy, having the soil a buffer effect that attenuates the injuries to the root system (Zhang et al., 
2005). Furthermore, we have not detected significant changes in proline and salicylates in exudates from heat-stressed plants, suggesting that the growth promotion in this case is mediated by different mechanisms than in the case of exudates obtained from saltstressed Macrophylla plants (Fig. 8). This is also supported by the delay in the growth promotion induced by exudates from heat-stressed plants.

\section{Conclusions}

To conclude, this work reveals that citrus root exudates can modulate the growth of the rhizobacteria $P$. putida KT2440 and Novosphingobium sp. HR1a, and it is affected by the genotype and abiotic stress conditions, promoting generally an increase of their growth when they are obtained from 60 or $90 \mathrm{mM} \mathrm{NaCl}$, or $40^{\circ} \mathrm{C}$ stressed plants. Moreover, root exudates from plants subjected to stress conditions tend to induce the expression of putA and pahA genes in both bacterial strains, indicating that they are able to detect proline and salicylates present in root exudates. The concordance between $\beta$-galactosidase activity and proline and salicylates contents quantified by other methods reveals that $P$. putida KT2442 (pMIS5) and Novosphingobium sp. HR1a (pPAH) can be respectively used for the detection of proline and salicylates in root exudates. On the other hand, this work reveals the importance of root exudates in the growth of PGPRs, which could be used as a fertilizer to improve PGPR colonization and therefore trigger plant benefits, especially under abiotic stress conditions.

\section{References}

Ali, S.Z., Sandhya, V., Grover, M., Linga, V.R., Bandi, V., 2011. Effect of inoculation with a thermotolerant plant growth promoting Pseudomonas putida strain AKMP7 on growth of wheat (Triticum spp.) under heat stress. J. Plant Interact. 6, 239-246.

Badri, D.V., Vivanco, J.M., 2009. Regulation and function of root exudates. Plant. Cell Environ. 32, 666-681.

Bais, H.P., Weir, T.L., Perry, L.G., Gilroy, S., Vivanco, J.M., 2006. The role of root exudates in rhizosphere interactions with plants and other organisms. Annu. Rev. Plant Biol. 57, 233-266.

Bensalim, S., Nowak, J., Asiedu, S.K., 1998. A plant growth promoting rhizobacterium and temperature effects on performance of 18 clones of potato. Am. J. Potato Res. 
$75,145-152$.

Bertani, G., 1951. Studies on Lysogenesis I. The mode of phageliberation by lysogenic Eschericia coli. J. Bacteriol. 62, 293-300.

Cesco, S., Mimmo, T., Tonon, G., Tomasi, N., Pinton, R., Terzano, R., Neumann, G., Wisskopf, L., Renella, G., Landi, L., Nannipieri, P., 2012. Plant-borne flavonoids released into the rhizosphere: Impact on soil bio-activities related to plant nutrition. A review. Biol. Fertil. Soils 48, 123-149

Chiquito-Contreras, R.G., Osorio-Acosta, F., García-Pérez, E., Villanueva-Jiménez, J. A., Zulueta-Rodríguez, R., Castillo Rocha, D.G., 2012. Biofertilization with rhizobacteria and a consortium of arbuscular mycorrhizal fungi in citrus rootstocks. Trop. Subtrop. Agroecosystems 15, S72-S81.

Dardanelli, M.S., Manyani, H., González-Barroso, S., Rodríguez-Carvajal, M.A., GilSerrano, A.M., Espuny, M.R., López-Baena, F.J., Bellogín, R.A., Megías, M., Ollero, F.J., 2010. Effect of the presence of the plant growth promoting rhizobacterium (PGPR) Chryseobacterium balustinum Aur9 and salt stress in the pattern of flavonoids exuded by soybean roots. Plant Soil 328, 483-493.

Dardanelli, M.S., de Córdoba, F.J.F., Estévez, J., Contreras, R., Cubo, M.T., RodríguezCarvajal, M.A., Gil-Serrano, A.M., López-Baena, F.J., Bellogín, R., Manyani, H., Ollero, F.J., Megías, M., 2012. Changes in flavonoids secreted by Phaseolus vulgaris roots in the presence of salt and the plant growth-promoting rhizobacterium Chryseobacterium balustinum. Appl. Soil Ecol. 57, 31-38.

Dimkpa, C., Weinand, T., Asch, F., 2009. Plant-rhizobacteria interactions alleviate abiotic stress conditions. Plant, Cell Environ. 32, 1682-1694.

Doornbos, R.F., Geraats, B.P.J., Kuramae, E.E., Van Loon, L.C., Bakker, P.A.H.M., 2011. Effects of jasmonic acid, ethylene, and salicylic acid signaling on the rhizosphere bacterial community of Arabidopsis thaliana. Mol. Plant. Microbe. Interact. 24, 395-407.

Franklin, F.C., Bagdasarian, M., Bagdasarian, M.M., Timmis, K.N., 1981. Molecular and functional analysis of the TOL plasmid pWWO from Pseudomonas putida and cloning of genes for the entire regulated aromatic ring meta cleavage pathway. Proc. 
Natl. Acad. Sci. U. S. A. 78, 7458-62.

Goldman, E., Green, L.H., 2008. Practical Handbook of Microbiology, second ed.. Boca Ratón, Florida.

He, Y., Wu, Z., Tu, L., Shan, C., 2017. Effect of encapsulated Pseudomonas putida Rs198 strain on alleviating salt stress of cotton. J. Plant Nutr. 40, 1180-1189.

Kawasaki, A., Donn, S., Ryan, P.R., Mathesius, U., Devilla, R., Jones, A., Watt, M., 2016. Microbiome and exudates of the root and rhizosphere of Brachypodium distachyon, a model for wheat. PLoS One 11, e0164533.

Kejela, T., Thakkar, V.R., Patel, R.R., 2017. A novel strain of Pseudomonas inhibits Colletotrichum gloeosporioides and Fusarium oxysporum infections and promotes germination of Coffee. Rhizosphere 4, 9-15.

Khadeejath Rajeela, T.H., Gopal, M., Gupta, A., Bhat, R., Thomas, G.V., 2017. Crosscompatibility evaluation of plant growth promoting rhizobacteria of coconut and cocoa on yield and rhizosphere properties of vegetable crops. Biocatal. Agric. Biotechnol. 9, 67-73.

Kohler, T., Curty, L.K., Barja, F., Van Delden, C., Pechere, J.C., 2000. Swarming of Pseudomonas aeruginosa is dependent on cell-to-cell signaling and requires flagella and pili. J. Bacteriol. 182, 5990-5996.

Krishnan, R., Menon, R.R., Likhitha, Busse, H.J., Tanaka, N., Krishnamurthi, S., Rameshkumar, N., 2017. Novosphingobium pokkalii sp nov, a novel rhizosphereassociated bacterium with plant beneficial properties isolated from saline-tolerant pokkali rice. Res. Microbiol. 168, 113-121.

López-Climent, M.F., Arbona, V., Pérez-Clemente, R.M., Gómez-Cadenas, A., 2008. Relationship between salt tolerance and photosynthetic machinery performance in citrus. Environ. Exp. Bot. 62:176-184.

Lugtenberg, B.J.J., Kravchenko, L.V, Simons, M., 1999. Tomato seed and root exudate sugars: composition, utilization by Pseudomonas biocontrol strains and role in rhizosphere colonization. Environ. Microbiol. 1, 439-446.

Miller, J., 1972. Experiments in Molecular Genetics. Cold Spring Harbor, New York. 
Moe, L.A., 2013. Amino acids in the rhizosphere: From plants to microbes. Am. J. Bot. 100, 1692-1705.

Murashige, T., Skoog, F., 1962. A revised medium for rapid growth and bioassays with tobacco tissue cultures. Physiol. Plant. 15, 473-497.

Navarro, J.M., Pérez-Tornero, O., Morte, A., 2014. Alleviation of salt stress in citrus seedlings inoculated with arbuscular mycorrhizal fungi depends on the rootstock salt tolerance. J. Plant Physiol. 171, 76-85.

Neal, A.L., Ahmad, S., Gordon-Weeks, R., Ton, J., 2012. Benzoxazinoids in root exudates of maize attract Pseudomonas putida to the rhizosphere. PLoS One 7, e35498.

Planchamp, C., Glauser, G., Mauch-Mani, B., 2015. Root inoculation with Pseudomonas putida KT2440 induces transcriptional and metabolic changes and systemic resistance in maize plants. Front. Plant Sci 5, 719.

Rietz, D.N., Haynes, R.J., 2003. Effects of irrigation-induced salinity and sodicity on soil microbial activity. Soil Biol. Biochem. 35, 845-854.

Rodríguez, A.A., Stella, A.M., Storni, M.M., Zulpa, G., Zaccaro, M.C., 2006. Effects of cyanobacterial extracellular products and gibberellic acid on salinity tolerance in Oryza sativa L. Saline Systems 2, 7.

Sakai, H., Hisamoto, S., Fukutomi, I., Sou, K., Takeoka, S., Tsuchida, E., 2004. Detection of lipopolysaccharide in hemoglobin-vesicles by Limulus amebocyte lysate test with kinetic-turbidimetric gel clotting analysis and pretreatment of surfactant. J. Pharm. Sci. 93, 310-321.

Satir, N. Y., Ortas, I., Satir, O., 2016. The influence of mycorrhizal species on sour orange (Citrus aurantium L.) growth under saline soil conditions. Pakistan J. Agric. Sci. 53, 399-406.

Segura, A., Hernández-Sánchez, V., Marqués, S., Molina, L., 2017. Insights in the regulation of the degradation of PAHs in Novosphingobium sp. HR1a and utilization of this regulatory system as a tool for the detection of PAHs. Sci. Total Environ. 590-591, 381-393. 
Spaink, H.P., Okker, R.J.H., Wijffelman, C.A., Pees, E., Lugtenberg, B.J.J., 1987. Promoters in the nodulation region of the Rhizobium leguminosarum Sym plasmid pRL1JI. Plant Mol. Biol. 9, 27-39.

Trivedi, P., Spann, T., Wang, N., 2011. Isolation and characterization of beneficial bacteria associated with citrus roots in Florida. Microb. Ecol. 62, 324-336.

Vílchez, S., Manzanera, M., Ramos, J.L., 2000a. Control of expression of divergent Pseudomonas putida put promoters for proline catabolism. Appl. Environ. Microbiol. 66, 5221-5225.

Vílchez, S., Molina, L., Ramos, C., Ramos, J.L., 2000b. Proline catabolism by Pseudomonas putida: Cloning, characterization, and expression of the put genes in the presence of root exudates. J. Bacteriol. 182, 91-99.

Vives-Peris, V., Gómez-Cadenas, A., and Pérez-Clemente, R.M., 2017. Citrus plants exude proline and phytohormones under abiotic stress conditions. Plant Cell Rep 36:1971-1984.

Watanarojanaporn, N., Boonkerd, N., Wongkaew, S., Prommanop, P., Teaumroong, N., 2011. Selection of arbuscular mycorrhizal fungi for citrus growth promotion and Phytophthora suppression. Sci. Hortic. (Amsterdam). 128, 423-433.

Widhalm, J.R., Dudareva, N., 2015. A familiar ring to it: Biosynthesis of plant benzoic acids. Mol. Plant 8, 83-97.

Wu, F., Wang, X., Xue, C., 2009. Effect of cinnamic acid on soil microbial characteristics in the cucumber rhizosphere. Eur. J. Soil Biol. 45, 356-362.

Wu, Q.S., Zou, Y.N., 2009. Mycorrhizal influence on nutrient uptake of citrus exposed to drought stress. Philipp. Agric. Sci. 92, 33-38.

Wu, Q.S., Zou, Y.N., 2010. Beneficial roles of arbuscular mycorrhizas in citrus seedlings at temperature stress. Sci. Hortic. (Amsterdam). 125, 289-293.

Yao, L., Wu, Z., Zheng, Y., Kaleem, I., Li, C., 2010. Growth promotion and protection against salt stress by Pseudomonas putida Rs-198 on cotton. Eur. J. Soil Biol. 46, $49-54$.

Zandalinas, S.I., Balfagón, D., Arbona, V., Gómez-Cadenas, A., 2017. Modulation of 
antioxidant defense system is associated with combined drought and heat stress tolerance in citrus. Front. Plant Sci. 8, Art. 953.

Zhang, L., Gao, J.S., Kim, S.G., Zhang, C.W., Jiang, J.Q., Ma, X.T., Zhang, J., Zhang, X.X., 2016. Novosphingobium oryzae sp. nov., a potential plant-promoting endophytic bacterium isolated from rice roots. Int. J. Syst. Evol. Microbiol. 66, 302307.

Zhang, N., Wang, D., Liu, Y., Li, S., Shen, Q., Zhang, R., 2014. Effects of different plant root exudates and their organic acid components on chemotaxis, biofilm formation and colonization by beneficial rhizosphere-associated bacterial strains. Plant Soil $374,689-700$.

Zhang, Y., Chen, W., Smith, S.L., Riseborough, D.W., Cihlar, J., 2005. Soil temperature in Canada during the twentieth century: Complex responses to atmospheric climate change. J. Geophys. Res. 110, D03112.

Zhang, Y.C., Wang, P., Wu, Q.H., Zou, Y.N., Bao, Q., Wu, Q.S. (2017). Arbuscular mycorrhizas improve plant growth and soil structure in trifoliate orange under salt stress. Arch. Agron. Soil Sci. 63, 491-500.

\section{Conflict of interest statement}

Authors declare that they have no competing interests.

\section{Authors and contributions}

VVP, RMPC and LM planned and designed the experiments. LM and AS aided in the interpretation of results. VVP obtained root exudates samples and realized the experiments with the bacterial strains. VVP and RMPC wrote the first draft of the manuscript and prepared the figures. AGC, LM and AS revised the subsequent revisions of the manuscript and prepared the final version. All authors have read and approved the final version of the manuscript. 


\section{Funding}

This work was supported by the Spanish Ministerio de Economia y Competitividad (MINECO) and Universitat Jaume I through grants AGL2016-76574-R and UJI-B201623 , respectively. VVP was recipient of a predoctoral contract from the Universitat Jaume I (PREDOC/2013/31). 
Table 1. Bacteria, plasmids and plants used. $\mathrm{Ap}^{\mathrm{r}}, \mathrm{Cm}^{\mathrm{r}}, \mathrm{Rif}^{\mathrm{r}}$ and $\mathrm{Tc}^{\mathrm{r}}$ refer to resistance to ampicillin, chloramphenicol, rifampicin and tetracycline respectively.

\begin{tabular}{|c|c|c|}
\hline $\begin{array}{c}\text { Bacterial strains, plasmids and } \\
\text { plant genotypes }\end{array}$ & Relevant characteristics & $\begin{array}{c}\text { Reference or } \\
\text { source }\end{array}$ \\
\hline \multicolumn{3}{|l|}{ Bacterial strains } \\
\hline P. putida $\mathrm{KT} 2440$ & Wild type; $\mathrm{Ap}^{\mathrm{r}}, \mathrm{Cm}^{\mathrm{r}}$ & Franklin et al. 1981 \\
\hline P. putida KT2442 & $\operatorname{Rif}^{\mathrm{T}}$ derivative of KT2440 & Franklin et al. 1981 \\
\hline Novosphingobium sp. HR1a & Wild type; $\mathrm{Tc}^{\mathrm{r}}$ & Segura et al. 2017 \\
\hline \multicolumn{3}{|l|}{ Plasmids } \\
\hline pMIS5 & $\mathrm{Tc}^{\mathrm{r}}, P_{\text {put }}:$ : ‘lac $Z$ or $\mathrm{RKK} 2$ & Vílchez et al. 2000 \\
\hline pMP220 & $\mathrm{Tc}^{\mathrm{r}}$,'lacZ ori $\mathrm{RK} 2$ & Spaink et al. 1987 \\
\hline pРAHa & $\mathrm{Tc}^{\mathrm{r}}, P_{\text {pahA }}:$ 'lacZ oriRK2 & Segura et al. 2017 \\
\hline \multicolumn{3}{|l|}{ Plant genotypes } \\
\hline Carrizo citrange & Salt sensitive, heat tolerant & Vives-Peris et al. 2017 \\
\hline Citrus macrophylla & Salt moderate tolerant, heat sensitive & Vives-Peris et al. 2017 \\
\hline
\end{tabular}

\section{FIGURE AND TABLE CAPTIONS}

Figure 1. Tolerance of $P$. putida KT2440 and Novosphingobium sp. HR1a to salt conditions. A: Turbidity at $\mathrm{OD}_{660 \mathrm{~nm}}$ of cultures of $P$. putida KT2440 and Novosphingobium sp. HR1a in presence of 0,60 and $90 \mathrm{mM} \mathrm{NaCl}$ in $\mathrm{M} 8$ medium plus $20 \mathrm{mM}$ succinate; white circles refer to control; black circles refer to $60 \mathrm{mM} \mathrm{NaCl}$ and black triangles refer to $90 \mathrm{mM} \mathrm{NaCl}$ treatments. B: Number of CFU of cultures of $P$. putida KT2440 and Novosphingobium sp. HR1a in presence of 0,60 and $90 \mathrm{mM} \mathrm{NaCl}$ in M8 medium plus $20 \mathrm{mM}$ succinate, after 6 hours; white bars refer to control at 0 hours; light grey, dark grey, and black bars refer to cultures in presence of 0,60 and $90 \mathrm{mM}$, respectively. Values indicate the mean of three replicates \pm standard error. Different letters to statistically significant differences at $\mathrm{P} \leq 0.05$.

Figure 2. Effect of root exudates from Carrizo citrange (A and C) and C. macrophylla (B and D) plants subjected to salt stress in the growth of P. putida KT2440 (A and B) and Novosphingobium sp. HR1a (C and D) in M8 medium plus $20 \mathrm{mM}$ succinate. White circles refer to control; black circles and black triangles refer to root exudates from plants subjected to 60 and $90 \mathrm{mM} \mathrm{NaCl}$, respectively. Values indicate the mean of three replicates \pm standard error.

Figure 3. Effect of root exudates from Carrizo citrange and C. macrophylla salt-stressed plants in the increase of the number of colony forming units of P. putida KT2440 and Novosphingobium sp. HR1a in M8 medium with $20 \mathrm{mM}$ succinate after 6 hours. White bars refer to control; grey bars refer to cultures with root exudates from $60 \mathrm{mM} \mathrm{NaCl}$ stressed plants, and black bars refer to cultures with root exudates from $90 \mathrm{mM} \mathrm{NaCl}$ stressed plants. Values are normalized according to root fresh weight and indicate the mean of three replicates \pm standard error. Different letters to statistically significant differences at $\mathrm{P} \leq 0.05$.

Figure 4. Effect of root exudates from Carrizo citrange (A and C) and C. macrophylla (B and D) plants subjected to heat stress in the growth of $P$. putida KT2440 (A and B) and Novosphingobium sp. HR1a (C and D) in M8 medium with $20 \mathrm{mM}$ succinate. White circles refer to control; black circles and black triangles refer to root exudates from plants 
subjected to $30{ }^{\circ} \mathrm{C}$ and $40{ }^{\circ} \mathrm{C}$, respectively. Values indicate the mean of three replicates \pm standard error.

Figure 5. Effect of root exudates from Carrizo citrange and C. macrophylla heat-stressed plants in the increase of the number of colony forming units of P. putida KT2440 and Novosphingobium sp. HR1a in M8 medium with $20 \mathrm{mM}$ succinate after 24 hours. White bars refer to control; grey bars refer to cultures with root exudates from $30{ }^{\circ} \mathrm{C}$ stressed plants, and black bars refer to cultures with root exudates from $40{ }^{\circ} \mathrm{C}$ stressed plants. Values are normalized according to root fresh weight and indicate the mean of three replicates \pm standard error. Different letters to statistically significant differences at $\mathrm{P} \leq$ 0.05 .

Figure 6. $\beta$-Galactosidase activity of cultures of $P$. putida KT2442 (pMIS5) and Novosphingobium sp. HR1a (pPAH) with different commercial standards. A: $\beta$ Galactosidase activity of cultures of $P$. putida KT2442 (pMIS5) supplemented with LProline (L-Pro) and L-Hydroxyproline (L-Hyp). B: $\beta$-Galactosidase activity of cultures of Novosphingobium sp. HR1a (pPAH) supplemented with methyl salicylate (Me-SA), sodium salicylate (Na-SA), L-phenylalanine (Phe), sodium benzoate (Na-Benz), pcoumaric acid ( $\mathrm{p}$-coum) and $t$-cinnamic acid (t-cin). Commercial standards were added at concentrations of $0.1 \mathrm{mM}$ (white bars) and $1 \mathrm{mM}$ (grey bars), and $\beta$-galactosidase activity was measured after 7 (non-lined bars) and 24h (lined bars). Values are referred to mock culture and indicate the mean of three replicates \pm standard error.

Figure 7. $\beta$-Galactosidase activity of P. putida KT2442 (pMIS5) and Novosphingobium sp. HR1a (pPAH) grown with root exudates from citrus plants subjected to salt stress for 10 days. $\beta$-Galactosidase activity was measured at 7 hours. White bars refer to control; grey bars refer to $60 \mathrm{mM} \mathrm{NaCl}$, and black bars refer to $90 \mathrm{mM} \mathrm{NaCl}$. Values indicate the mean of three replicates \pm standard error.

Figure 8. $\beta$-Galactosidase activity of P. putida KT2442 (pMIS5) and Novosphingobium sp. HR1a (pPAH) grown with root exudates from citrus plants subjected to heat stress for 10 days. $\beta$-Galactosidase activity was measured at 24 hours. White bars refer to control, grey bars refer to $30^{\circ} \mathrm{C}$, and black bars refer to $40^{\circ} \mathrm{C}$. Values indicate the mean of three replicates \pm standard error.

Table 1. Bacteria, plasmids and plants used. $\mathrm{Ap}^{\mathrm{r}}, \mathrm{Cm}^{\mathrm{r}}, \operatorname{Rif}^{\mathrm{r}}$ and $\mathrm{Tc}^{\mathrm{r}}$ refer to resistance to ampicillin, chloramphenicol, rifampicin and tetracycline respectively.

Sup. Figure 1. Salicylic acid biosynthesis and conjugation in plants. Adapted from Widhalm and Dudareva 2015. 
Figure 1
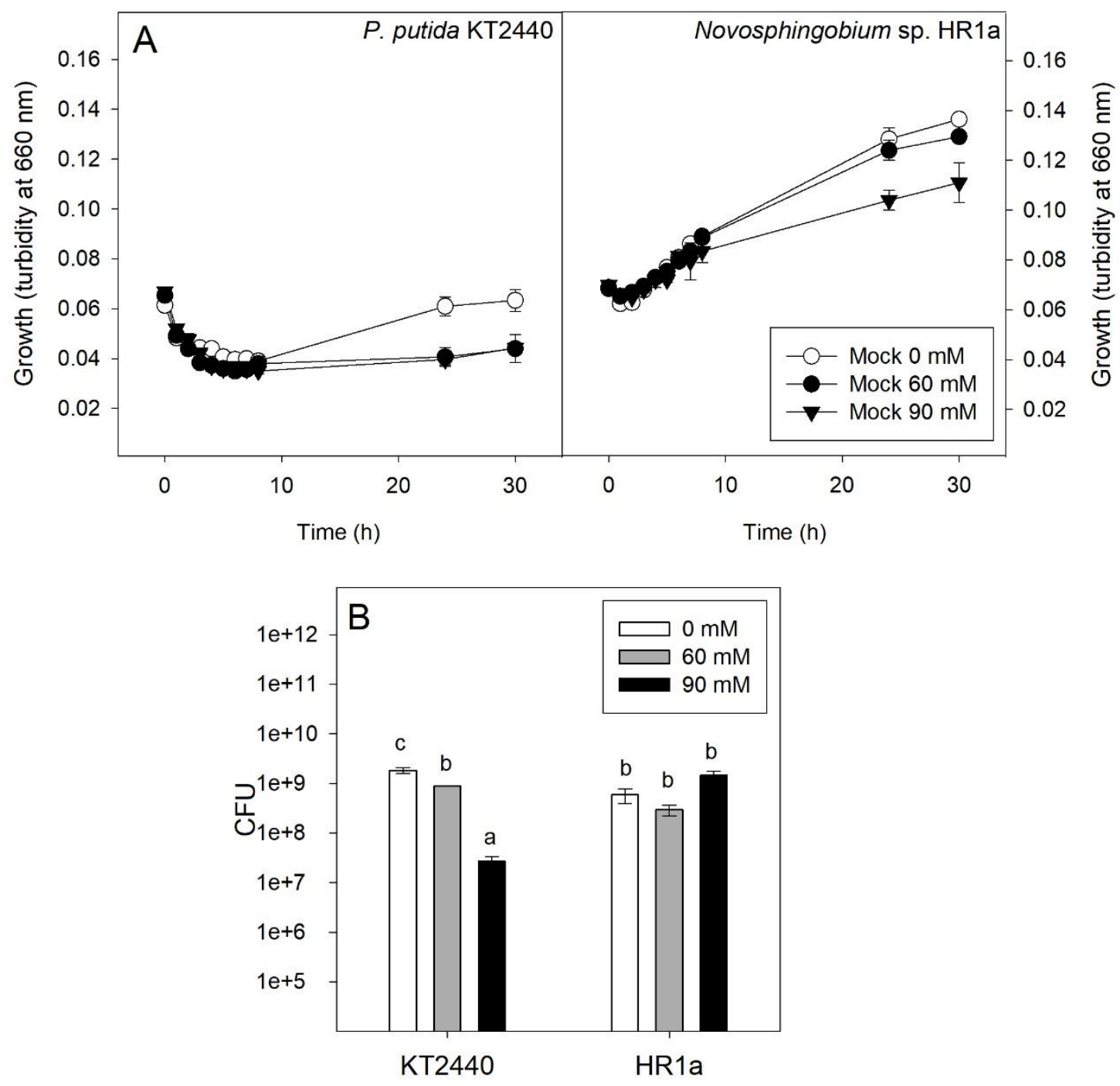
Figure 2

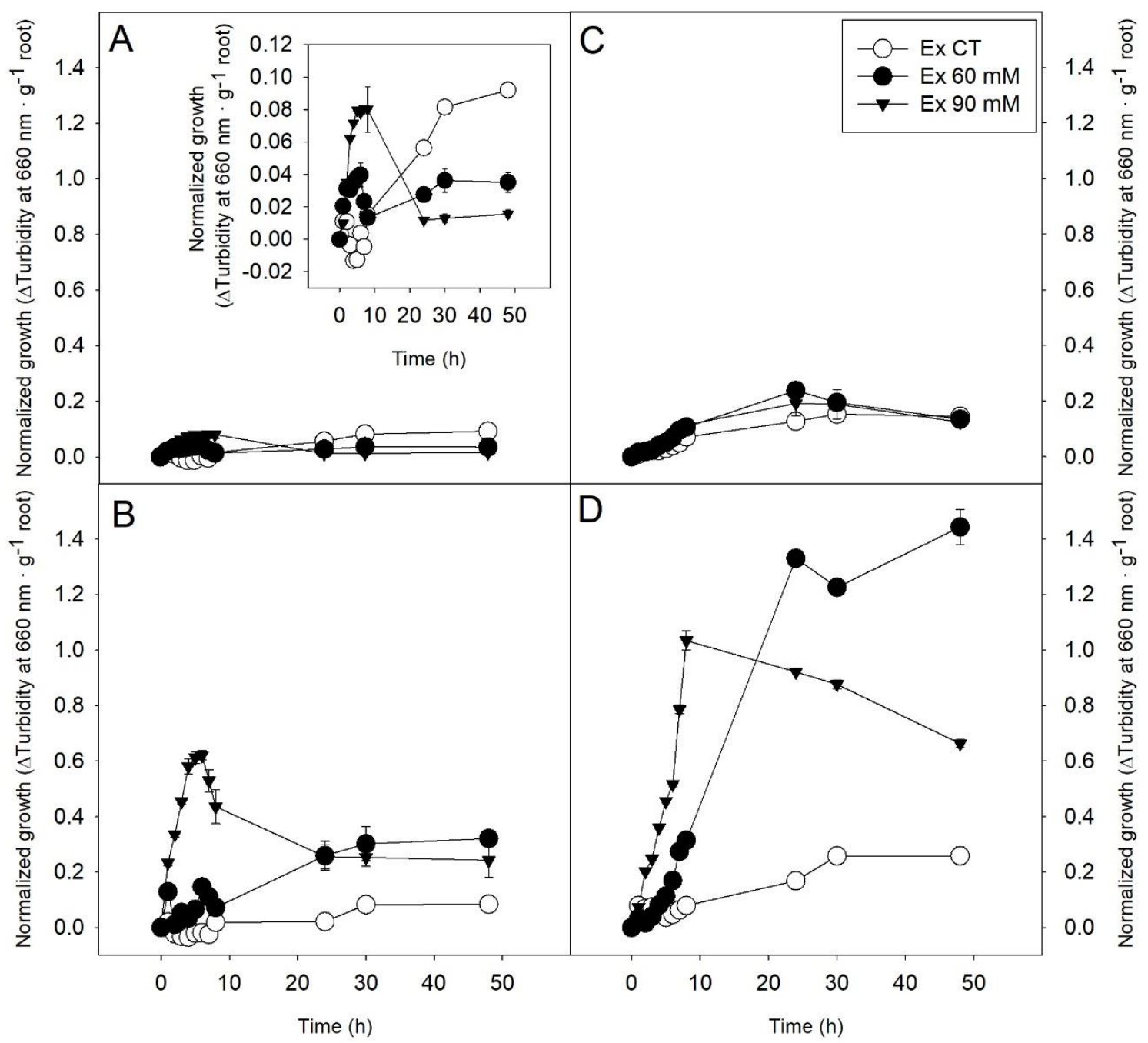

Figure 3

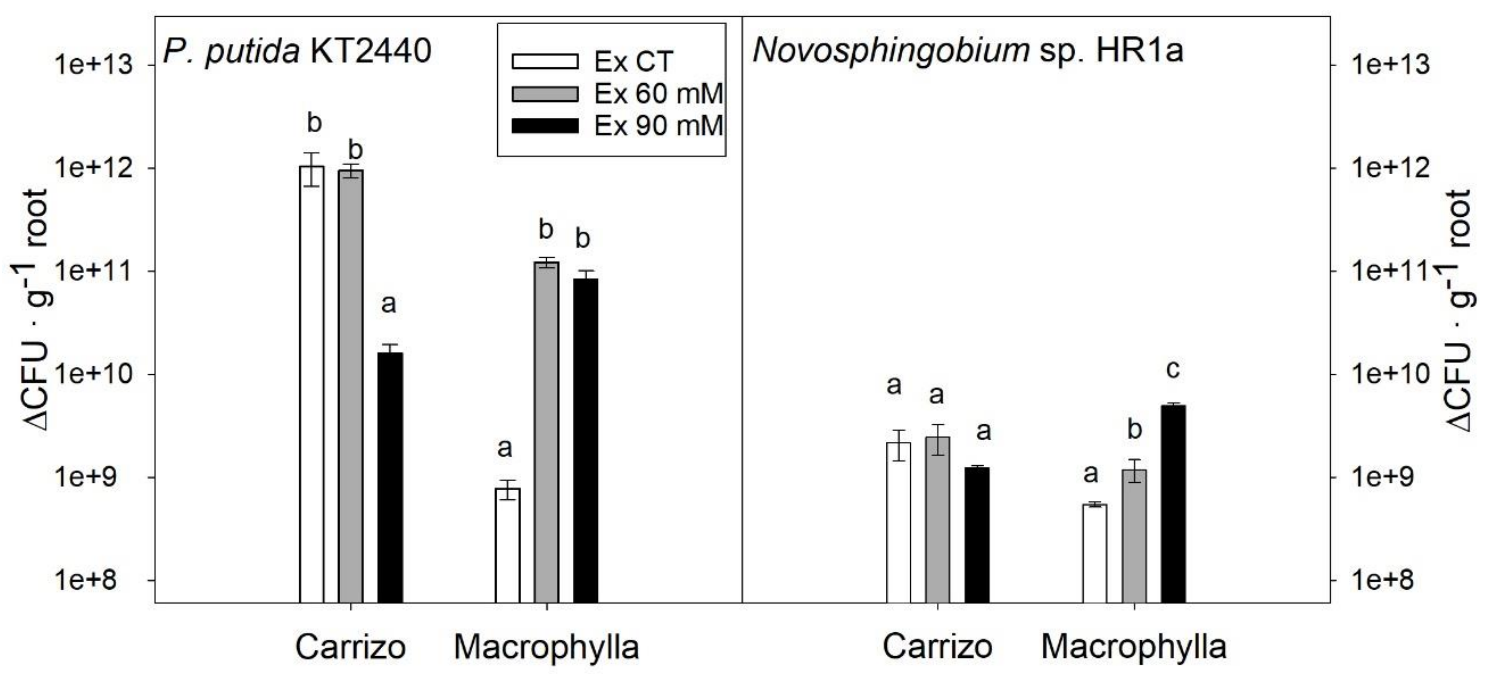


Figure 4

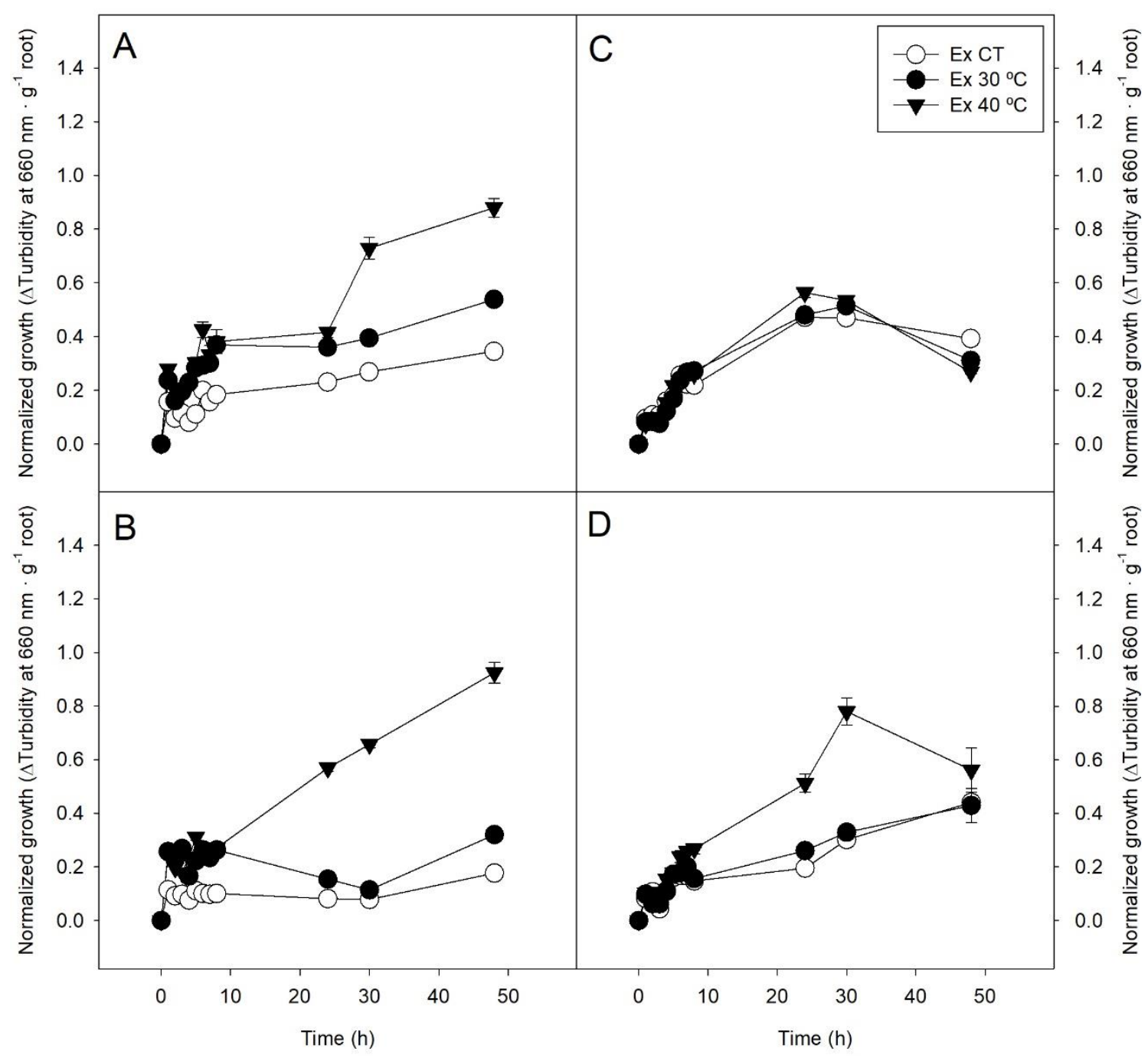

Figure 5

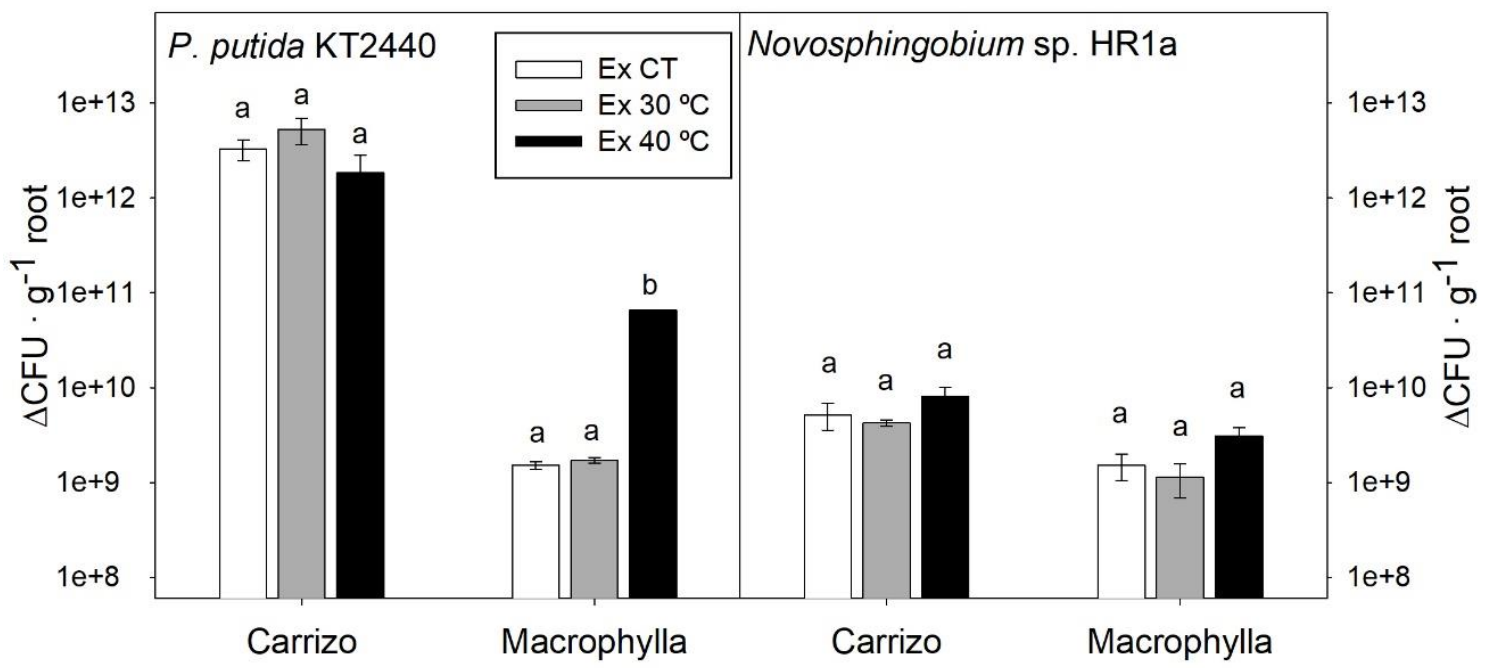


Figure 6
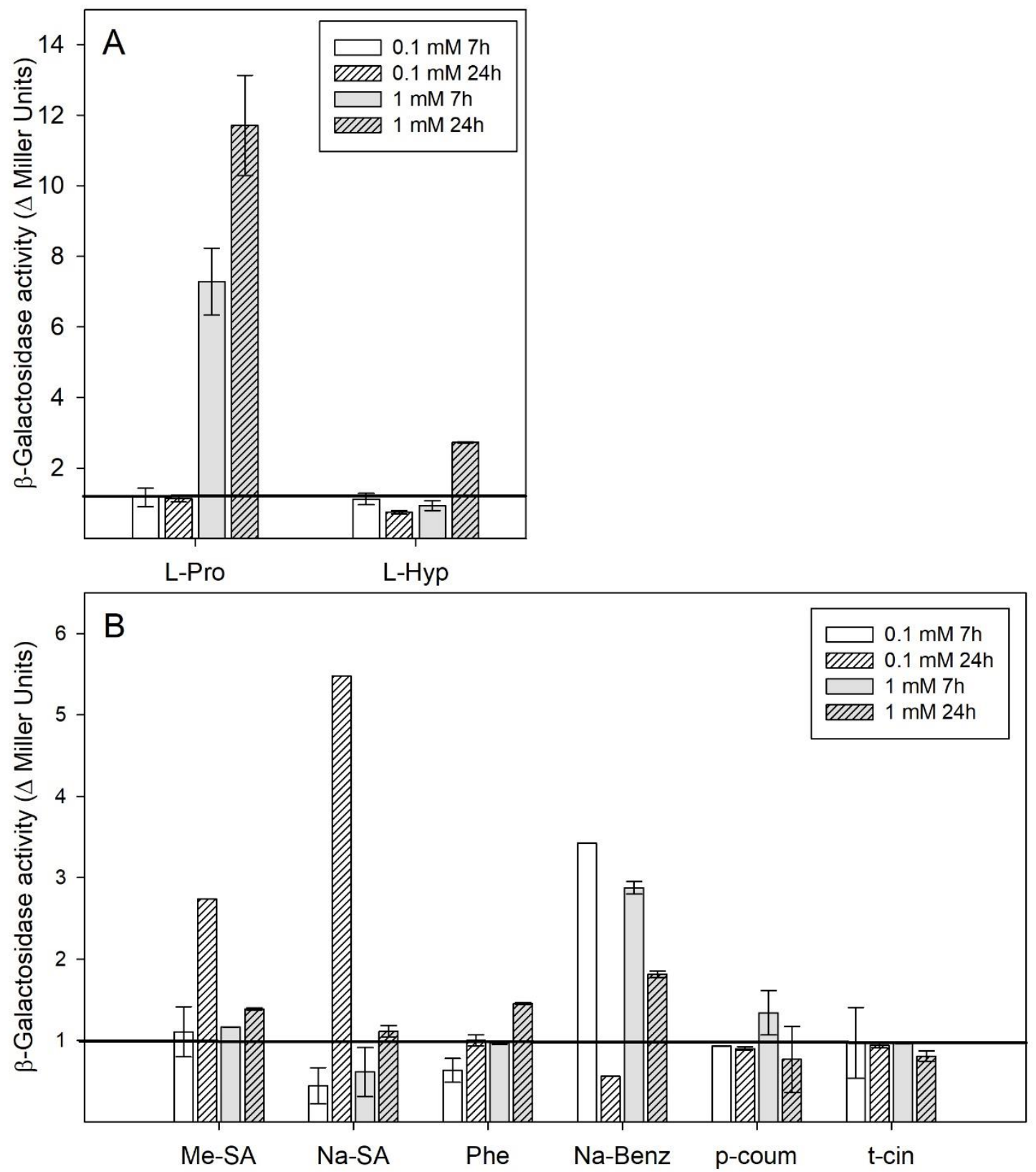
Figure 7

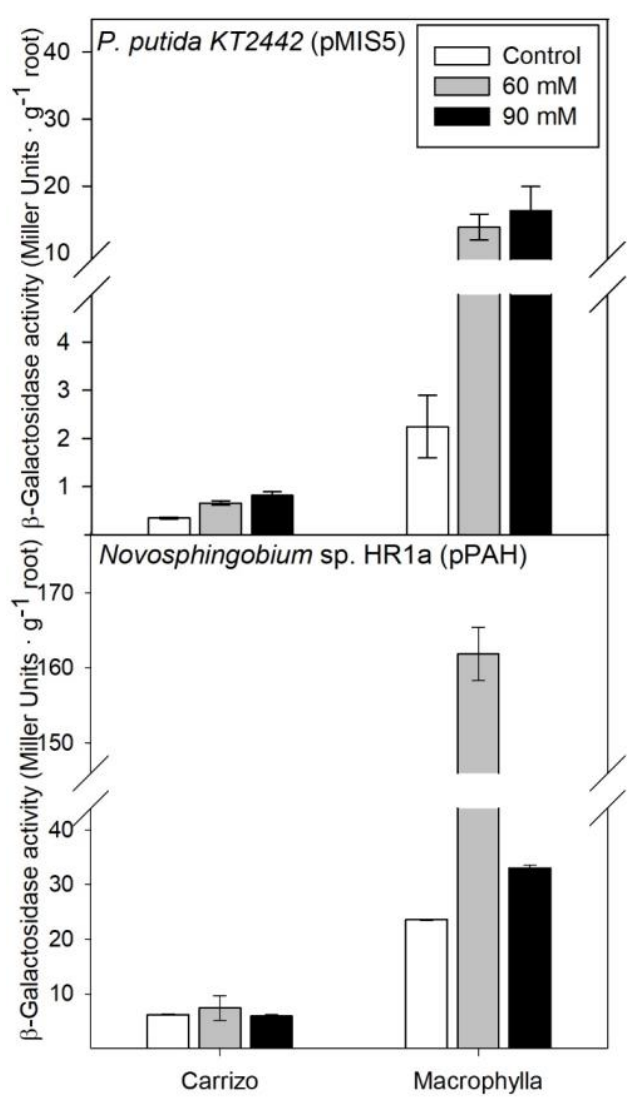

Figure 8

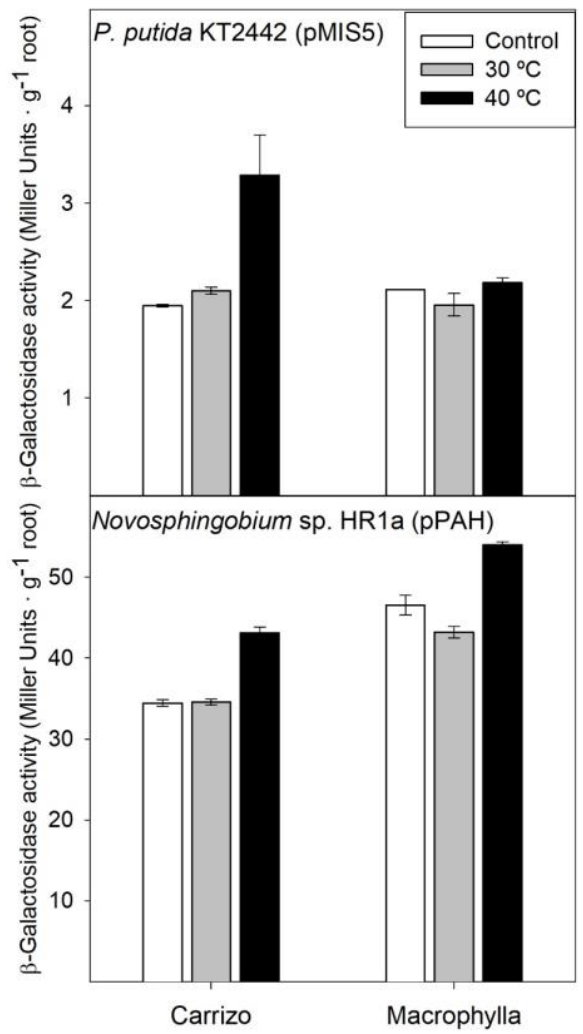

